\title{
Blueprint on Comparison of WSN Protocols
}

\author{
Prof. Mayuri Bapat \\ MIT ACSC, ALANDI(D), Pune
}

\begin{abstract}
A sensor network is a group of specialized devices that converts energy of one form into another that also designed to monitor and record condition at places of different areas. Commonly monitored parameters are humidity, temperature, air pressure, wind speed and direction, sunlight, volume intensity, vibration intensity, illumination intensity, pollution levels and Human Emotions etc. This paper provides the general idea of WSN, Unique features of WSN, WSN Node Architecture and Routing protocols of Sensor Network.

Keywords: Wireless Sensor Network, Topology, MANET, Routing Protocols
\end{abstract}

\section{Introduction To Wireless Sensor Networks:-}

A sensor network consists of sensor nodes which work as multiple detection stations. Each node is tiny, frivolous and movable unit. Every sensor node is consist of a microcomputer, transceiver, transducer and power source. The physical effects get sensed and electrical signals get generated by transducer. Generated sensor output get stored by microcomputer processes. Generally hard-wired or some wireless devices are worked as transceiver and transmits data to other computer system. Battery or electric service derives power for each sensor.

Applications of sensor networks include:

- In Business

- In Class Room

- For Safety and Security i.e. video inspection

- For Environment i.e. monitoring and sensing of climate conditions

- In Medical Field, for monitoring of medical devices

- In automation of Industrial tools

- Automated and smart homes

- In Traffic monitoring

- In Robotics

- In Aeronautical Science

\section{Inimitable Features Of Sensor Networks:-}

It is obvious that ad hoc network and sensor network have some common things to share.

Hence, protocols designed for sensor networks must comprise for the properties of wireless (ad-hoc) networks, including the subsequent properties.

1. Untrustworthy data transmission because of wireless network.

2. Though WSN having lot of unique features which are not present in existing ad-hoc networks, there is need of self configuration of nodes which requires slight human involvement.

3. As network consists of nodes and nodes get charged by either electrical supply or batteries, hence every node is having limited life.

4. Number of nodes in WSN is more than traditional ad-hoc networks.

5. Traditional nodes provided mobility and hence every node is over weighted, but sensor nodes are usually motionless.

6. Due to harsh environment, node failure is common.

7. As node size of sensor network is small they are facing challenges like short lifetime, less memory and calculation power.

8. Wireless sensor network requires location info.

9. Sensor networks are more cooperative than traditional wireless network as sensor networks try to achieve similar worldwide aim which helps to maintain quality of service.

10. As sensor network uses M-to-1 traffic, "Wi-Fi" problems may occur.

11. Packets are used to move the data and hence layer wise overhead get increased.

Including these exclusive features, Sensor network capably make use of bounded resources of network. This escorts to quite different protocols for different layers of network, each with 
This has led to quite a number of protocols from the data-link layer up to the transport layer, each with the aim of permit the network to operate separately for as long as possible while maintaining network processing and data channels to give the application's essential quality of service.

\section{Wireless Sensor Networks Architecture:-}

Following topologies are used for communication in WSN. Following is the brief discussion of different topologies used in WSN.

- Point-to-point

- Star Topology

- Mesh Topology

- Hybrid Topology

- Tree Topology

Point-to-point Topology [5]:-

In Point-to-point topology, wireless link is present between two sensor nodes. This is dedicated link for these two nodes i.e. other than two connected nodes no other node able to transfer data. This is one of the basic traditional topology.

Advantage:-

- It provides security

- It provides fast data transfer

\section{Disadvantage:-}

- If link fails, data transfer not possible
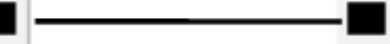

Fig.a: Point-to-point topology

\section{Star Network (Single Point-to-Multipoint):-}

In Star topology communication is made using single centralized base node, it means base node can send and receive messages to other remote nodes. Remote nodes are only capable to receive messages or send messages to/from central node only. They cannot communicate with remote nodes directly.

\section{Advantage:-}

- It reduces power utilization of remote nodes which automatically reduces charging cost.

- This topology is scalable i.e. we can easily add or remove nodes.

- It reduces data transfer delays.

Disadvantage:-

- If central node fails, communication is not possible

- Base node should me in the range of other remote nodes.

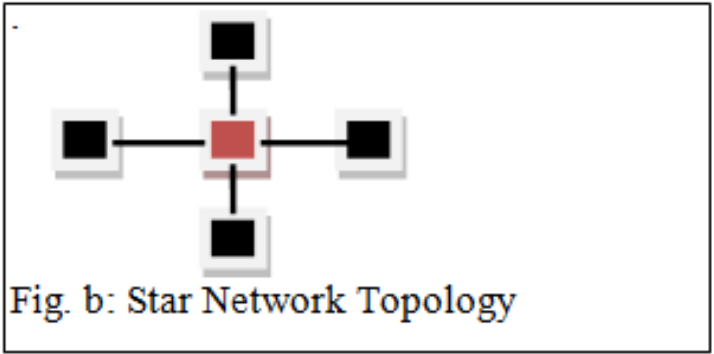

Mesh Network:-

In mesh network, any one node can transmit the data directly to any other node available in network. Mesh topology supports hopping concept to transfer the data, if a destination node is not a neighboring node, then to transfer data source node uses nodes which are in-between source and destination node. 
Advantages:-

- If any node fails, other nodes can able to send the messages to desired node.

- It is a scalable network.

- It also supports redundancy.

Disadvantages:-

- Nodes used in mesh topology require more power consumption because no node is in idle state.

- Due to support of hopping concept, message requires more time to deliver.

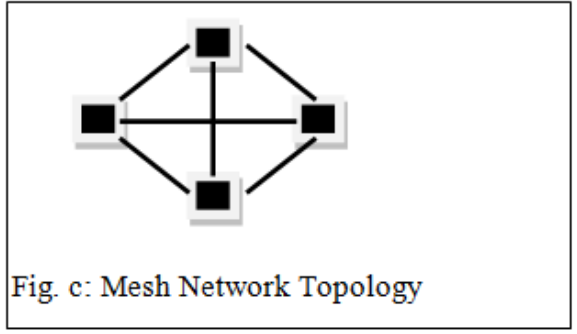

\section{Hybrid Network:-}

Hybrid topology consists of star and mesh network gives vigorous and flexible network for communication, whilst keeping less power consumption of sensor nodes. In this topology, sensor nodes with low power are not able to forward the messages. Other sensor nodes are capable to transfer or forward the messages with multi hopping facility to other nodes in the network. To get higher power, sensor nodes are frequently plugged into main line of electricity. "ZigBee" implements this topology.

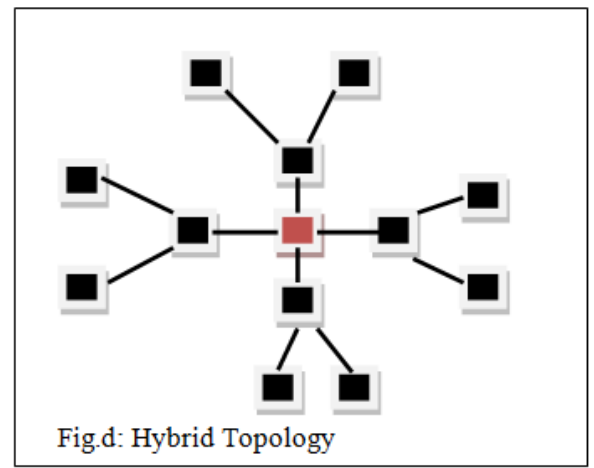

\section{Routing Protocols For Sensor Networks:-}

Traditional routing protocols are implemented on routers for successful data transmission over the network. In the same way to transfer data in wireless network, WSN protocols are used.

In this section, we present an overview of routing protocols designed for WSN, starting with network characteristics and designed objectives, network design challenges and routing issues followed by routing protocols in WSN that we were able to study. This study is by no means exhaustive.

\section{1 (Shio Kumar Singh, 2010)}

Shio Kumar Singh, M.P.Singh \& D.K.Singh presents the concept of network characteristics, network design objectives, network design challenges and routing issues for a wireless sensor network. Following points illustrate their concepts.

\subsubsection{Network Characteristics}

As per the study of unequaled [1], following are the characteristics \& constraints of WSN which have to consider during design of WSN.

- Dense sensor node deployment

- Battery powered sensor node

- Severe energy, computation and storage

- Constraints, self configurability, unreliable

- data duplication, application 
- Specific , many to one traffic pattern

- Frequent topology changes

\subsubsection{Network Design Objectives}

Design of sensor network changes as per the application. Thus some or all of the following design objectives should get considered during design of WSN.

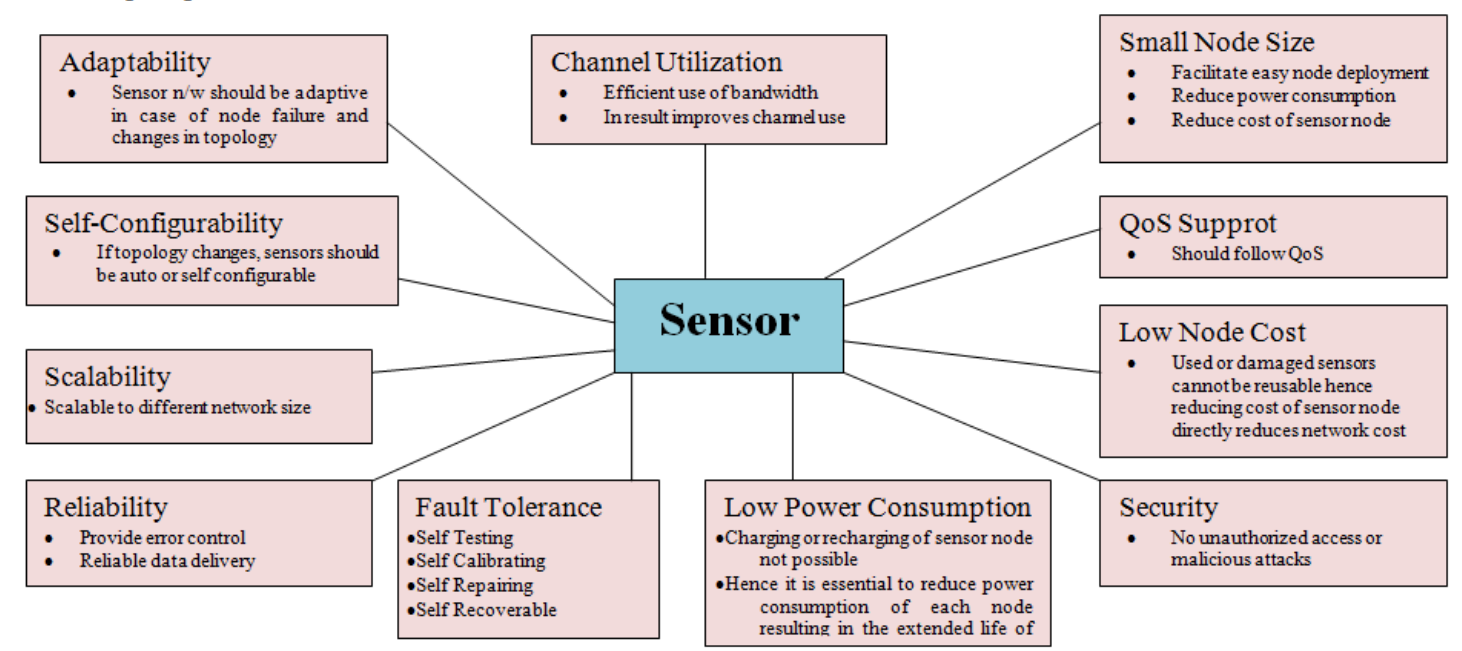

Fig. 1 Network Design Objectives

\subsubsection{Network Design Challenges \& Routing Issues}

As we know WSN have limitations of bandwidth, energy, storage and processing unit. Following are the main aspects in design challenges of WSN[1].

- Limited Energy Capacity: - As sensors batteries are not chargeable, all WSN suffer through problem of limited energy capacity. Secondly sensor become faulty once it reaches a certain threshold. Hence WSN protocols should be energy thorough as possible to increase their life.

- Sensor locations and limited hardware resources: - Routing protocols should be able to manage sensor locations. As we know, sensors have limited energy, besides that it have limited processing and storage capacity.

- Massive and random node deployment: - Sensor node deployment is either manual or random and application specific which affects routing protocol performance.

- Characteristics of network and unreliable environment: - Topology of the network vary due to node failure or node addition in the network. All nodes are connected using wireless medium which is generally noisy and error prone hence such $\mathrm{n} / \mathrm{w}$ is unreliable and random.

- Data Aggregation: - It is required to reduce number of redundant data transmissions. This technique achieves energy efficiency and minimal data transfer.

\subsubsection{Types of routing protocols: -}

WSN routing differs from traditional routing in fixed network in various ways. In WSN, no infrastructure installed, links are unreliable, sensor node failure and protocol have to meet energy saving requirement. Many routing algorithms were developed for wireless networks in general and may be divided into seven different categories[1] as shown in table 1.

\begin{tabular}{|l|l|}
\hline Category & Representative Protocols \\
\hline Location-based Protocols & MECN, SMECN, GAF, GEAR, Span, TBF, BVGF, GeRaF \\
\hline Data-centric Protocols & $\begin{array}{l}\text { SPIN, Directed Diffusion, Rumor Routing, COUGAR, ACQUIRE, EAD, Information-Directed } \\
\text { Routing, Gradient- Based Routing, Energy-aware Routing, Information-Directed Routing, } \\
\text { Quorum-Based Information Dissemination, Home Agent Based Information Dissemination }\end{array}$ \\
\hline Hierarchical Protocols & LEACH, PEGASIS, HEED, TEEN, APTEEN \\
\hline Mobility-based Protocols & $\begin{array}{l}\text { SEAD, TTDD, Joint Mobility and Routing, Data MULES, } \\
\text { Dynamic Proxy Tree-Base Data Dissemination }\end{array}$ \\
\hline Multipath-based Protocols & $\begin{array}{l}\text { Sensor-Disjoint Multipath, Braided Multipath, N-to-1 } \\
\text { Multipath Discovery }\end{array}$ \\
\hline Heterogeneity-based Protocols & IDSQ, CADR, CHR \\
\hline QoS-based protocols & SAR, SPEED, Energy-aware routing \\
\hline
\end{tabular}

Table 1: Routing Protocols for WSNs 


\subsubsection{Location Based Protocols:}

In Location based protocols(LBP), sensor nodes are addressed via their locations. Most of routing protocols use location information for finding the distance between two nodes so that energy consumption can be calculated. Geographic Adaptive routing(GAF) and Span protocols are mainly designed for MANET but also used for WSNs because their aim is to condensed energy consumption of the nodes. They based on the mechanism of turning off sensor nodes when they are idle. Design of GAF considers energy required due to packet receive and transmission.

Some LBPs are hardware equipped to find the location e.g. GPS to know their up to date position and to estimate distance from their neighbouring sensor e.g. GEAR \& TBF.

\subsubsection{Data Centric Protocols:}

To control the redundancy of data, Data Centric Protocols are used. Data redundancy occurs because sensor node does not have global recognition number which identifies them uniquely, so data is communicated to each node with considerable redundancy. In this protocol sink sends request for the data, the sink request for data by sending the query hence nearest sensor pass on the data selected understand from the query. The property of data is specified by attribute based naming.

Flooding and gossiping: These two are the mechanisms works without using routing algorithms and topology maintenance to transmit the data.

Flooding: Sensor node broadcasts the data to its neighbors till the packet arrive at the destination [14].

Gossiping: In this, data packet is send to the arbitrarily chosen neighbor which decides on another random neighbor to promote the data and so on. Its benefit is that it keep away from implosion. However this cause delay in propagation of data among nodes.[1][8]

Implosion: It is due to facsimile messages send to same neighbor node,

Overlap problem: Duplicate data can be received by the nodes because one sensor may be in range of more than one network. This area network is called as overlapping region.

Resource blindness: Available energy of the nodes does not consider by flooding protocol which results in duplicate message sending. Therefore it diminishes lifetime of network.

\subsubsection{Hierarchical Protocols:}

These protocols save total energy utilization of the network in WSN. Hierarchy is getting followed in each clusters having head node. Head node having tasks like data collection and data aggregation from neighbouring clusters and send that data to Base Station. This aggregation reduces energy utilization in the network as less number of data messages transferred to the base station; hence it directly increases network life. It increases operating cost of the network because of configuration of the cluster and the cost required for its maintenance. But it gives best performance as compare to other topologies of network.

\subsubsection{Mobility Based Protocols:}

Due to the supremacy of obstacles, mobile sensor nodes change their locations after its deployment. Mobility results numerous changes in the in network topology which initiates the problems which are mentioned below:-

- Mobility escort to decline in the quality of an established link hence results in data failure which increases rate of retransmission of packet.

- Packet delivery delay occur because of mobility as node changes their locations very frequently.

- Mobile node cannot start data sending immediately after change in location because neighbor node first determine its presence and come to a decision of how to work together. This needs a few time.

\subsubsection{Multipath Routing Protocols:}

The multipath routing technique used to discover alternate pathway between source and sink which gives provides efficiency to get better performance of wireless sensor. This approach overcomes the limitations of routing [5].

Benefits of Multipath Routing:

- Reliability and Fault-Tolerance

- Load Balancing

- QoS Improvement

- Reduced Delay

- Bandwidth Aggregation

Elements of Multipath Routing Protocol are:

- Path discovery

- Traffic distribution

DOI: 10.9790/0661-180405122129 
- Path maintenance

\subsubsection{Heterogeneity Based Protocols:}

Line-powered sensors and Battery powered sensors are used in Heterogeneity Based Protocols. In Line-powered sensors energy consumption have no limits. On other side battery powered sensors have energy constraint. In heterogeneity based protocols, these two protocols together extend sensor life which straightly rises network lifetime.

\subsubsection{QoS Based Protocols:-}

Qos Based protocols give surety quality of service along with minimum energy consumption. It offers QoS in terms of reliability, fault tolerance and delay of data transfer in WSN. Sequential Assignment Routing(SAR) ,SPEED and Energy-Aware QoS Routing Protocols that assist in finding a balance between energy utilization and QoS demands.

\section{2 (Rajashri V. Biradar)}

Rajashri V. Biradar, V.C. Patil, Dr. S.R.Sawant \& Dr.R R Mudholkar presents the concept of Introduction to Sensors, MANETS and sensor network, Comparison of routing protocols etc. Following points illustrates their concepts.

\subsubsection{Introduction}

Sensors are mainly used for sensing expected events, data processing. Sensors also work as communication components. In Sensor network, each node consist of Sensor Unit, Central Processing Unit, Power Unit and Communication unit. Each unit having different task.

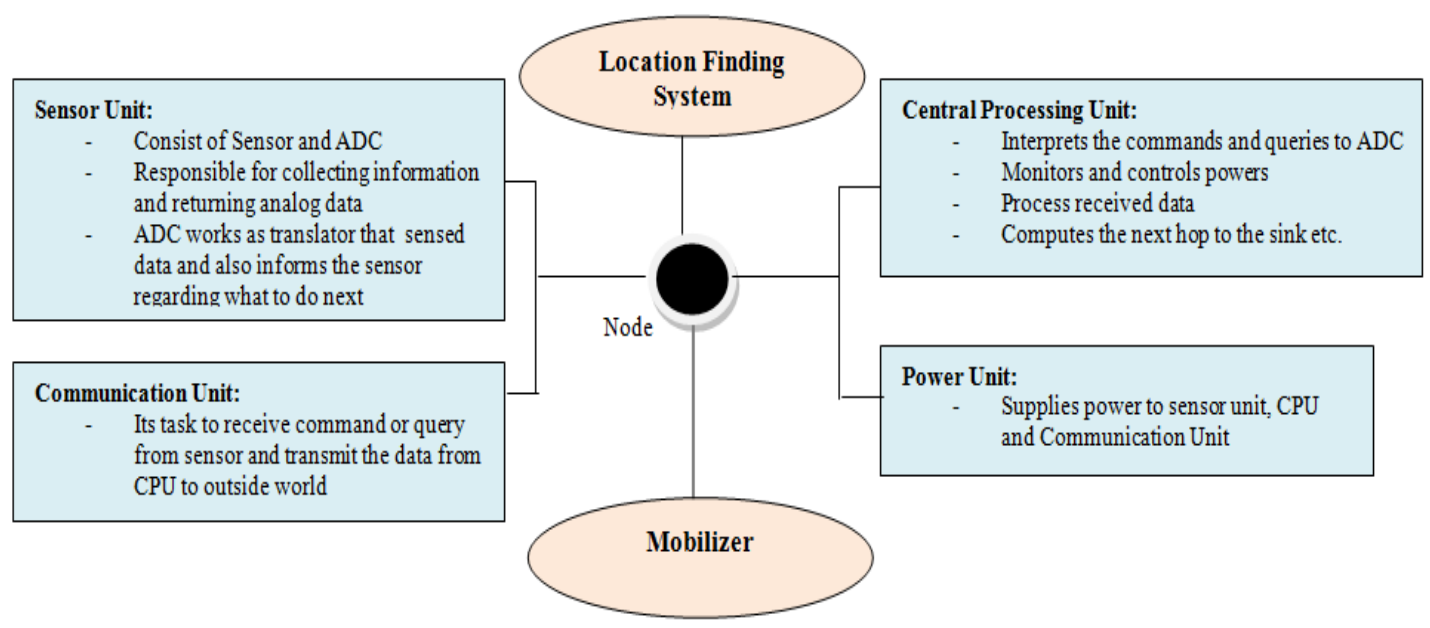

Fig. 2: Units of Sensor Network

\subsubsection{Difference of MANETS and Sensor Network}

MANETS(Mobile Ad hoc Net) and Wireless Sensor Network are two types of Wireless Ad hoc network. Each having its own resource limitations. Following is the comparison between them.

\begin{tabular}{|l|l|}
\hline Sensor Networks & MANETS \\
\hline Mainly used to collect information & Designed for distributed computing \\
\hline Uses broadcast communication & Uses point-to-point communication \\
\hline Number of nodes are more & Less number of nodes \\
\hline May not have global identification & Have global identification \\
\hline Cheaper than nodes in MANETS & Not cheaper \\
\hline Limited in power, computational capacities and memory & Nodes may recharged some time \\
\hline Nodes deployed once in their lifetime & Nodes move in Ad hoc maaner \\
\hline Less powerful & More powerful \\
\hline
\end{tabular}

\subsubsection{Comparison of Routing Protocols}

Comparison of routing protocols based on their design characteristics are given below[7]. This study is done by author[7], we used it for study purpose only. 
Blueprint on Comparison of WSN Protocols

\begin{tabular}{|c|c|c|c|c|c|c|c|c|}
\hline $\begin{array}{l}\text { Routing } \\
\text { Protocols }\end{array}$ & Classification & $\begin{array}{l}\text { Power } \\
\text { Usage }\end{array}$ & $\begin{array}{l}\text { Data } \\
\text { Aggregation }\end{array}$ & $\begin{array}{l}\text { Scala } \\
\text { Bility }\end{array}$ & $\begin{array}{l}\text { Query } \\
\text { Based }\end{array}$ & $\begin{array}{l}\text { Over } \\
\text { Head }\end{array}$ & $\begin{array}{l}\text { Data delivery } \\
\text { Model }\end{array}$ & QoS \\
\hline SPIN & $\begin{array}{l}\text { Flat / Srcinitiated } \\
\text { /Data- centric }\end{array}$ & Ltd. & Yes & Ltd & Yes & Low & Event driven & No \\
\hline DD & $\begin{array}{l}\text { Flat/ Datacentric/ } \\
\text { Dstinitiated }\end{array}$ & Ltd. & Yes & Ltd & Yes & Low & Demand Driven & No \\
\hline RR & Flat & Low. & Yes & Good & Yes & Low & Demand Driven & No \\
\hline GBR & Flat & Low. & Yes & Ltd & Yes & Low & Hybrid & No \\
\hline CADR & Flat & Ltd & & Ltd & Yes & Low & Continuously & No \\
\hline COUGAR & Flat & Ltd & Yes & Ltd & Yes & High & Query driven & No \\
\hline ACQUIRE & Flat/ Datacentric & Low. & Yes & Ltd & Yes & Low & Complex Query & No \\
\hline LEACH & $\begin{array}{l}\text { Hierarchical / } \\
\text { Dst-initiated } \\
\text { /Node-centric }\end{array}$ & High & Yes & Good & No & High & Cluster- head & No \\
\hline $\begin{array}{l}\text { TEEN \& } \\
\text { APTEEN }\end{array}$ & Hierarchical & High & Yes & Good & No & High & Active threshold & No \\
\hline PEGASIS & Hierarchical & Max & No & Good & No & Low & Chains Based & No \\
\hline VGA & Hierarchical & Low & Yes & Good & No & High & Good & No \\
\hline SOP & Hierarchical & Low & No & Good & No & High & Continuously & No \\
\hline GAF & $\begin{array}{l}\text { Hierarchical / } \\
\text { Location }\end{array}$ & Ltd & No & Good & No & Mod & Virtual Grid & No \\
\hline SPAN & $\begin{array}{l}\text { Hierarchical / } \\
\text { Location }\end{array}$ & Ltd & Yes & Ltd & No & High & Continuously & No \\
\hline GEAR & Location & Ltd & No & Ltd & No & Mod & Demand Driven & No \\
\hline SAR & Data Centric & High & Yes & Ltd & Yes & High & Continuously & Yes \\
\hline SPEED & $\begin{array}{l}\text { Location/Data } \\
\text { centric }\end{array}$ & Low & No & Ltd & Yes & Less & Geographic & Yes \\
\hline
\end{tabular}

\section{3(Luis Javier Garcia Villalba)}

Luis Javier Garcia Villalba, Ana Lucila Sandoval Orozco, Alicia Trivino Cabrera \& Claudia Jacy Barenco Abbas presents the concept of Routing Protocols In WSN which focused on Introduction, Algorithm Paradigms for WSn, Design Constraints for Routing in WSN and Routing Protocols proposed by Spanish Universities etc. Following is the illustration of their concepts.

\subsubsection{Algorithm Paradigms for WSN}

IN WSN, communication is done by using algorithms. Following diagram shows three types of algorithm.

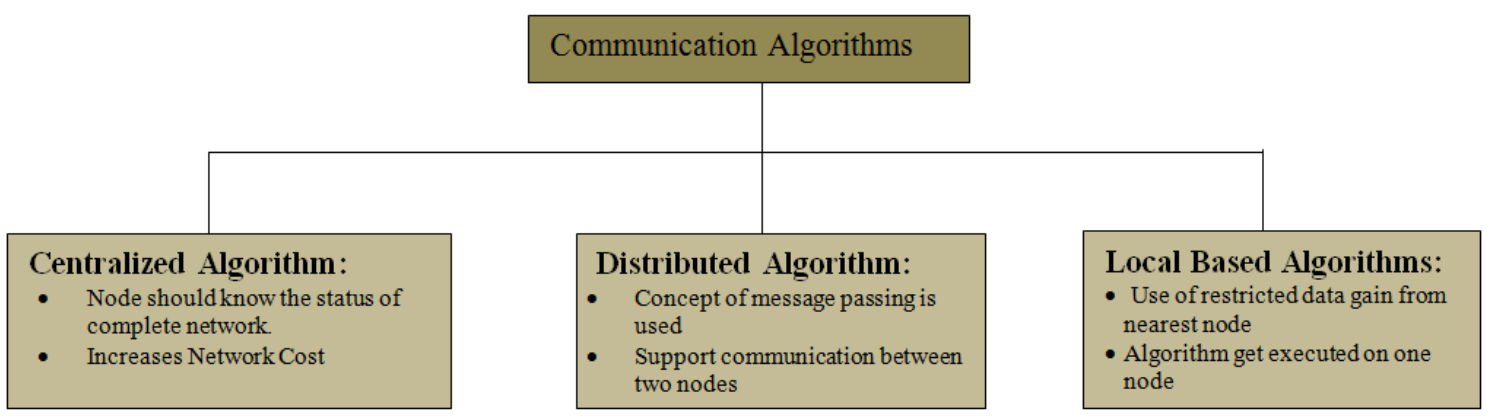

Fig.3: Algorithm Paradigms for WSN

\subsubsection{Constraints of Routing in WSN}

Following requirements should be accomplished by routing protocols in WSN.

1. self-government

2. Energy competence

3. Node Heterogeneity

4. Mobility Compliance

\subsubsection{Routing protocols recommended by Spanish University:}

a) Beacon-less Geographic Routing Protocols:

Here, beacons mean messages. Additional overhead occurs on network as intervallic exchange of beacons which informs node positions in the network. This algorithm reduces network overhead because location get exchanged only if nodes want to route data. Following are the five beaconless protocols.

- Implicit Geographic Forwarding(IGF)

- Geographic Random Forwarding (GeRaF) 
- $\quad$ BLR

- Contention Based Forwarding

- BOSS

\section{b) QoS Routing Protocols based on Artificial Intelligence}

It supports implementation of Neural Network into sensors and self organized map is used in the network. It reduces delay and overload of network as compared to Direct Diffusion Protocol.

\section{c) Energy Aware Routing Protocols for Underwater Sensor Networks}

In Underwater sensor networks, to interact with nodes and for data collection sensors are used. There are huge chances of propagation delay and errors because of severe change in network dynamics. Energy consumption varies depending on shallow water and deep water scenario. Direct communication gives nastiest performance.

\section{d) SHRP(Simple Hierarchical Routing Protocol)}

SHRP introduces new metric called hops. With the help of this metric SHRP can decide a route which considers number of hops to sink node but it selects the route having less number of hops. There are three types intellecting data messages.

- Periodic data messages: sent after specific periodic time.

- Alert data messages:- sent when sensed data value is above average value

- Alarm data messages:- sent when sensed data value is below a minimum or above a threshold value

\section{Conclusion}

This research work give highlights on WSN architecture, Network objectives, routing protocols and routing protocol algorithms. It also gives review of three research papers which gave detail knowledge about WSN and its protocols.

\section{References}

[1]. Wireless Sensor Networks by C. S. Raghavendra, Krishna M. Sivalingam and Taieb Znati

[2]. Communication Protocols for Sensor Networks by Weilian Su, Ozgur Baris Akan, and Erdal Cayirci

[3]. Energy Efficient hardware design by Vijay Raghunathan, Curt Schurgers, Sung Park and Mani Srivastava

[4]. "Insights to Sensor Technology and it's Applications" by Prof. Mayuri Bapat

[5]. "Wireless Sensor Networks- A Review on Topologies and Node Architecture" by Shamneesh Sharma, Dinesh Kumar and Keshav Kishore

[6]. 24.pdf

[7]. Classification and comparison of routing protocols in wireless sensor networks by Rajashree.V.Biradar , V.C .Patil , Dr. S. R. Sawant , Dr. R. R. Mudholkar

[8]. "Routing Protocols in Wireless Sensor Networks" by Luis Javier Garcia Villalba, Ana Lucila Sandoval Orozco, Alicia Trivino Cabrera \& Claudia Jacy Barenco Abbas. ISSN: 1424-8220

[9]. "A Survey on sensor networks" by Ian F. Akyildiz, Weilian Su, Yogesh Sankaraubramaniam, and Erdal Cayirci: IEEE Communications Magazine (2002)

[10]. "SPAN: an Energy-efficient Coordination Algorithm for Topology Maintenance in Ad Hoc Wireless Networks"; by B.Chen et al., vol. 8, no. 5, pp. 481-94, (Sept. 2002).

[11]. Geographical and Energy-Aware Routing: A Recursive Data Dissemination Protocol for Wireless Sensor Networks, by Y. Yu, D. Estrin, and R. Govindan,UCLA Computer Science Department Technical Report, UCLA-CSD TR-01-0023 ( May 2001). 